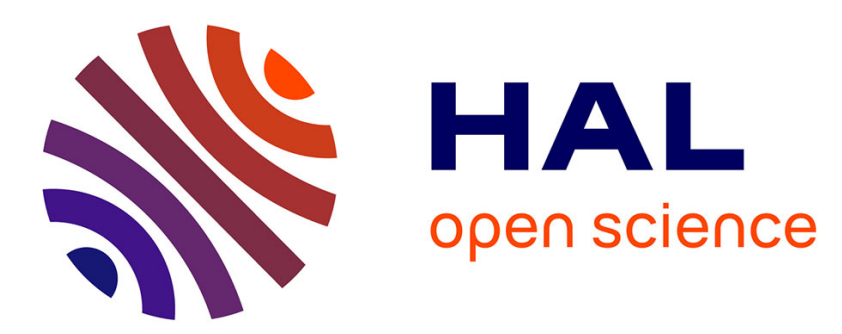

\title{
Organic Liquid Crystals as Single-Ion Li + conductors
}

Dominic Bresser, Mélody Leclere, Laurent Bernard, Patrice Rannou, Hakima

Mendil-jakani, Guk-tae Kim, Tatiana Zinkevich, Sylvio Indris, Gérard Gebel, Sandrine Lyonnard, et al.

\section{- To cite this version:}

Dominic Bresser, Mélody Leclere, Laurent Bernard, Patrice Rannou, Hakima Mendil-jakani, et al.. Organic Liquid Crystals as Single-Ion Li + conductors. ChemSusChem, 2021, 14 (2), pp.655-661. 10.1002/cssc.202001995 . hal-03413220

\section{HAL Id: hal-03413220 \\ https://hal.science/hal-03413220}

Submitted on 5 Nov 2021

HAL is a multi-disciplinary open access archive for the deposit and dissemination of scientific research documents, whether they are published or not. The documents may come from teaching and research institutions in France or abroad, or from public or private research centers.
L'archive ouverte pluridisciplinaire HAL, est destinée au dépôt et à la diffusion de documents scientifiques de niveau recherche, publiés ou non, émanant des établissements d'enseignement et de recherche français ou étrangers, des laboratoires publics ou privés. 


\section{Organic Liquid Crystals as Single-lon $\mathrm{Li}^{+}$Conductors}

Dominic Bresser ${ }^{+},[a, c, d]$ Mélody Leclere ${ }^{+},{ }^{[b]}$ Laurent Bernard, ${ }^{[a]}$ Patrice Rannou, ${ }^{[a]}$ Hakima Mendil-Jakani, ${ }^{[a]}$ Guk-Tae Kim, $\left.{ }^{[c,}, d\right]$ Tatiana Zinkevich, ${ }^{[c, ~ e] ~ S y l v i o ~ I n d r i s, ~}{ }^{[c,}$ e] Gérard Gebel, ${ }^{[b]}$ Sandrine Lyonnard, ${ }^{*}[a]$ and Lionel Picard*[b]

[a] Dr. D. Bresser,+ Dr. L. Bernard, Dr. P. Rannou, Dr. H. Mendil-Jakani, Dr. S. Lyonnard

Univ. Grenoble Alpes, CEA, CNRS, IRIG-SyMMES 38000 Grenoble (France)

E-mail: sandrine.lyonnard@cea.fr

[b] Dr. M. Leclere, ${ }^{+}$Dr. G. Gebel, Dr. L. Picard

Univ. Grenoble Alpes, CEA, Liten 38000 Grenoble (France)

E-mail: lionel.picard@cea.fr

[c] Dr. D. Bresser,+ Dr. G.-T. Kim, Dr. T. Zinkevich, Dr. S. Indris

Helmholtz Institute Ulm (HIU) Helmholtzstrasse 11, 89081 Ulm (Germany)

[d] Dr. D. Bresser,+ Dr. G.-T. Kim

Karlsruhe Institute of Technology (KIT) P.O. Box 3640, 76021 Karlsruhe (Germany)

[e] Dr. T. Zinkevich, Dr. S. Indris

Institute for Applied Materials - Energy Storage Systems (IAM-ESS), KIT Hermann-von-Helmholtz-Platz 1, 76344

Eggenstein-Leopoldshafen (Germany)

$[+]$ These authors contributed equally to this work.

Abstract: The development of new materials for tomorrow's electrochemical energy storage technologies, based on thoroughly designed molecular architectures is at the forefront of materials research. In this line, we report herein the development of a new class of organic lithium-ion battery electrolytes, thermotropic liquid crystalline single-ion conductors, for which the single-ion charge transport is decoupled from the molecular dynamics (i.e., obeys Arrhenius-type conductivity) just like in inorganic (single-)ion conductors. Focusing on an in-depth understanding of the structure-to-transport interplay and the demonstration of the proof-of-concept, we provide also strategies for their further development, as illustrated by the introduction of additional ionic groups to increase the charge carrier density, which results in a substantially enhanced ionic conductivity especially at lower temperatures.

Keywords: organic liquid crystal, conduction mechanism, decoupling, electrolyte, lithium battery

\section{Introduction}

Electrochemical energy storage in batteries is presently considered key for the successful transition to renewable energy sources and $\mathrm{a} \mathrm{CO}_{2}$-free transportation.[1-3] To reach this desirable, though highly challenging goal, further advances are needed. Polymer electrolytes are contemplated the pivotal near-future technology for enabling lithiummetal batteries, which are essential for achieving further increased energy densities.[4-7] Nevertheless, one remaining roadblock is their limited ionic conductivity due to its dependency on the segmental relaxation of the cationcoordinating polymer chains, i.e., their isotropic 'liquid-like' conduction behaviour, eventually hampered by the high viscosity of the polymer.[8,9] Accordingly, it has been proposed that the key to overcome this intrinsic conductivity limitation would be to decouple charge transport and segmental motion and, by this, realize a 'solid-like' charge transport.[10-14] Till date, this was partially realized for nanostructured crystalline poly(ethylene oxide),[15,16] "frustrated" fragile polymers,[17] polymerized ionic liquids, [18] or liquid crystalline (macro-)molecules,[19-23] comprising a lithium salt and, for higher conductivities, also a highly dielectric liquid phase. However, the recorded conductivity values are largely reflecting the anionic motion.[24] Here, we show that the selective cation conduction in solvent-free, soft, and easily processable organic electrolytes can be decoupled by realizing thermotropic liquid crystalline single-ion conductors (TLCSICs). These liquid crystalline molecules self-organize with increasing temperature into layered supramolecular self-assemblies with well-defined one-dimensional lithium-ion conduction pathways for which the hopping distance is defined by the $\pi-\pi$ interaction of promesogenic aromatic moieties. Remarkably, this new generation of smart organic electrolytes is very stable in contact with metallic lithium, allowing for an effective, constant-current lithium transport for more than $150 \mathrm{~h}$, while the 'solid-like' ionic conductivity of the "baseline molecule" can be increased by ca. three orders of magnitude via doubling the charge-carrier density through introducing a second ionic group onto the aromatic moiety.

\section{Results and Discussion}

\section{Design of thermotropic liquid crystalline single-ion conductors}

The generic design rule for TLCSICs is summarized in Figure 1a. Four key features need to be realized: A cationic charge carrier, a covalently bonded anionic function ensuring the single-ion conductivity, a promesogenic group with a pronounced intermolecular interaction potential, and a flexible long-chain spacer allowing for self-assembling nanostructures toward well-defined ion transport pathways. Following these considerations, we synthesized a series of molecules (Figure 1b) bearing a naphthalene unit as a promesogenic aromatic core with potential for $\pi$ $\pi$ interactions,[25] a covalently bonded sulfonate anionic function, a lithium cation as the charge carrier, and 1,4butanediol diglycidyl ether (BDGE) [1] or poly(dimethylsiloxane) diglycidyl ether (PDMSDGE) [2] as the non-polar flexible longchain spacer. The general synthesis protocol is depicted in Figure 1c. We will refer to these TLCSICs hereinafter as ANLi-BDGE and ANLi-PDMSDGE. 
(a)

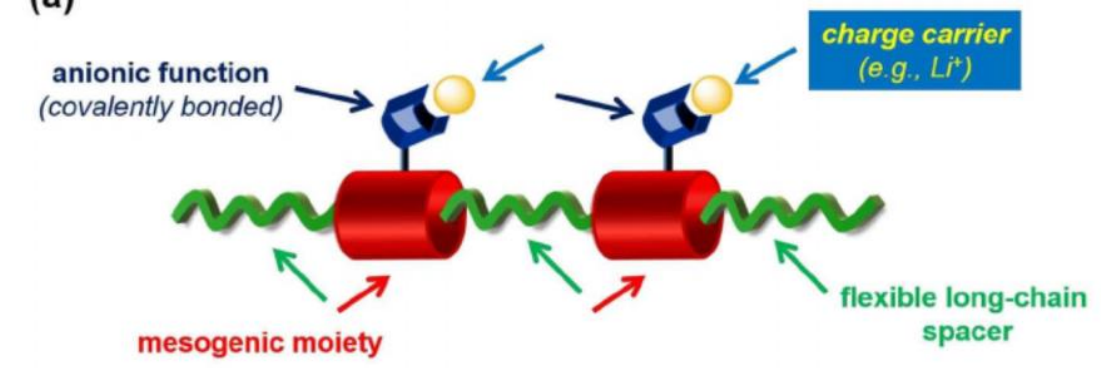

(b)
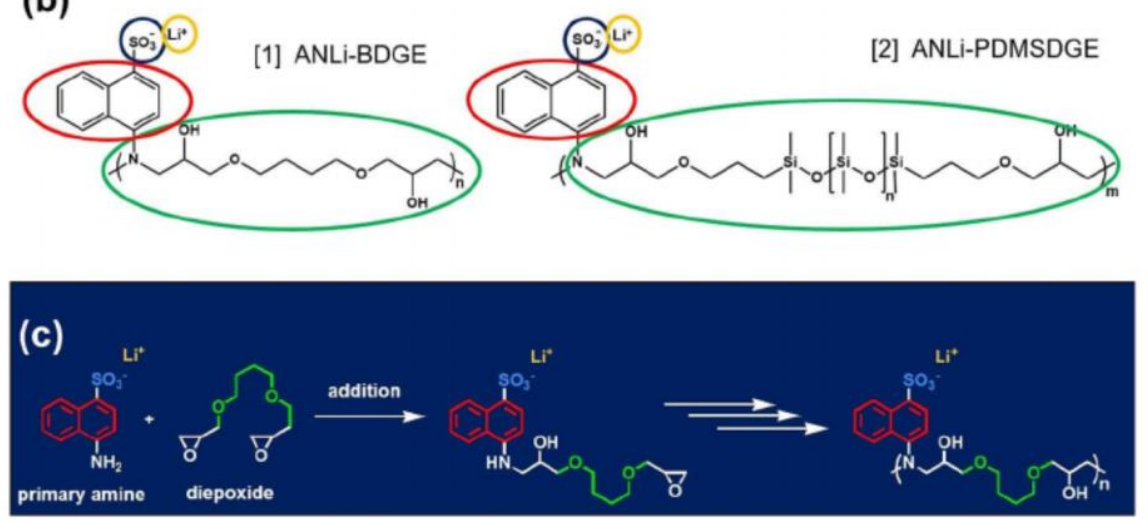

Figure 1. (a) Representation of the generic design principles towards TLCSICs. (b) Two examples for the herein reported TLCSICs, bearing these four functional features as indicated by the corresponding color coding. (c) The general synthesis protocol in brief, starting from a primary amine (e.g., lithium-4-amino-1-napthalenesulfonate (ANLi)) and diepoxide derivative (e.g., 1,4butanediol glycidyl ether (BDGE [1]) or poly(dimethylsiloxane)diglycidyl ether (PDMSDGE [2]), reacting by a simple addition reaction. The successful synthesis has been confirmed by ${ }^{1} \mathrm{H}$ and ${ }^{13} \mathrm{C}$ nuclear magnetic resonance as well as Fourier transform infrared spectroscopic characterization (see Experimental and Supporting Information, Table S1-S3 and Figure S1-S3).

\section{Liquid crystalline (nano-)structure}

In a first step, we studied the self-assembly of these TLCSICs by means of polarizing optical microscopy (POM), differential scanning calorimetry (DSC), and small-angle X-ray scattering (SAXS). Selected POM microphotographs for ANLi-BDGE are shown in Figure S4a (in the Supporting Information), revealing a transition from a glassy solidstate at room temperature to a rubbery phase at ca. $50{ }^{\circ} \mathrm{C}$. This perfectly agrees with the second order phase transition observed by DSC [i.e., the glass transition temperature $(\mathrm{Tg})$ ] at ca. $45^{\circ} \mathrm{C}$ related to the ether-based longchain spacer.[24,26] The optical extinction under cross-polarizers indicates the subsequent long range homeotropic alignment of a smectic-like mesophase, leaving only a few typical defects (Maltese crosses [27,28]) over areas of a few hundred $\mu \mathrm{m}^{2}$. Heating these TLCSICs above $210^{\circ} \mathrm{C}$ reveals the transition to an isotropic phase prior to the onset of their thermal decomposition occurring at ca. $250^{\circ} \mathrm{C}$ (Figure S5a). Such wide temperature range for a mesophase is common for thermotropic ionic liquid crystals (TILCs) owing to the long-range stabilizing effect provided by ionic interactions.[27] Here, this effect is even more pronounced due to the potential hydrogen-bonding interactions of the hydroxyl functions, located close to the naphthalene moiety.[29] Analogous results were observed for ANLiPDMSDGE (Figure 2a), revealing a transition to the rubbery phase at ca. $40{ }^{\circ} \mathrm{C}\left(\mathrm{Tg}=24^{\circ} \mathrm{C}\right)$, a slightly distorted alignment upon mechanical shearing as indicated by the colour change,[27] and a clearing point at ca. $235^{\circ} \mathrm{C}$. The lower rubbery phase transition and $\mathrm{Tg}$ as well as the marginally lower thermal stability (slightly below $250^{\circ} \mathrm{C}$; Figure $\mathrm{S5b}$ ) are assigned to the introduction of the highly flexible siloxane group.[30] Such high thermal stability presents a clear advantage compared to other organic electrolytes, requiring the blending with a conductive salt, which decomposes at temperatures as low as $60^{\circ} \mathrm{C}$ in case of LiPF6.[31] The variable temperature SAXS characterization of ANLi- BDGE and ANLi-PDMSDGE revealed similar features in both cases (Figure S4b and Figure 2a,b). We observed an initially rather broad ring at low temperatures for the 2D SAXS pattern, which gains in intensity and significantly narrows when increasing the temperature (Figure 2a), confirming a substantial increase in structural organization. Starting from ca. $110^{\circ} \mathrm{C}$, the transition to a smectic like lamellar mesophase is manifested by the appearance of first and second order rings2D/reflections1D at $q_{1} \sim 0.084 \AA^{-1}$ and $2 q_{1}$, respectively, as well as an indicated third-order peak $3 q_{1}$ (Figure $2 b$ ). The corresponding temperature-independent correlation distance is $d_{1}=74$ $\AA$ (i.e., twice the chain length). The simultaneous narrowing and increasing intensity of the lamellar peaks are associated with highly correlated hydrophobic regions with limited interdigitation of the chains. The lamellar ordering is maintained upon subsequent cooling. Additionally, two relatively broad reflections $q_{2}$ and $q_{3}$ are evidenced at around 0.54ANLi-BDGE/0.50ANLi- PDMSDGE $\AA^{-1}$ and $1.57 \AA^{-1}$, respectively. While the latter $\left(q_{3}\right)$ remains essentially unaffected when increasing the temperature, the former (q2) is significantly impacted and clearly shifts to lower $q$ values. This behavior translates for the corresponding correlation distances into an essentially temperatureindependent distance $d_{3}$ of ca. $4 \AA$, while $d_{2}$ is experiencing a shift from 11.6 to $14.3 \AA$ and from 12.6 to $14.9 \AA$ in case of ANLi-BDGE (Figure S4c) and ANLi- PDMSDGE (Figure 2c), respectively. Hence, we propose the following assignment for the two distances: $d_{3}$ corresponds to the $\pi$ - $\pi$ stacking developing in-between the naphthalene moieties and $d_{2}$ relates to the mean separation distance between the ionic channels; the latter one is linearly increasing with 
temperature for both TLCSICs, starting from ca. $110^{\circ} \mathrm{C}$ (Figure $2 \mathrm{c}$ and S4c; see also the extended discussion of the SAXS data in the Supporting Information). These observations are consistent with a self-assembly mechanism consisting of a temperature-induced $\pi-\pi$ stacking reinforced lamellar alignment.[27]

a

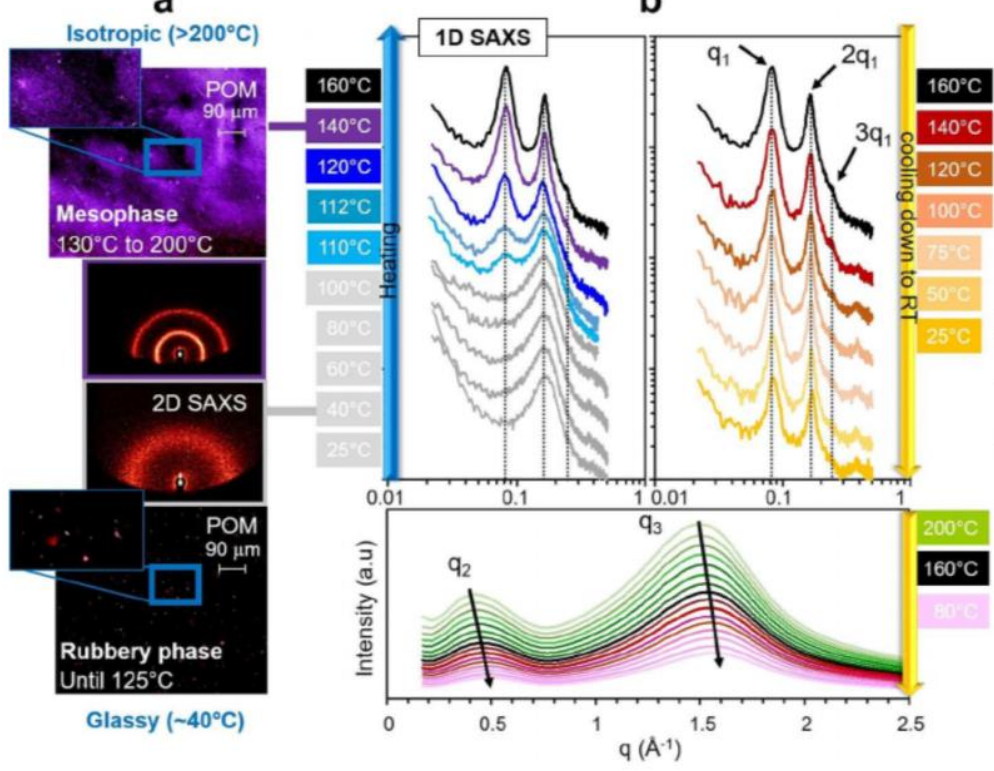

C

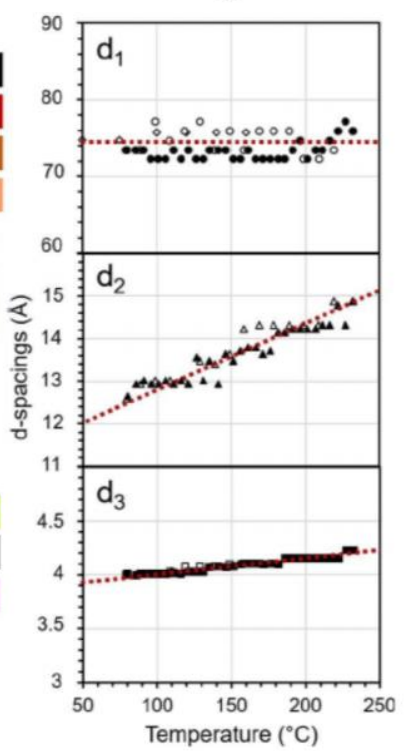

Figure 2. Characterization of ANLi-PDMSDGE by means of (a) polarizing optical microscopy (POM), indicating the temperaturedependent phase transitions and by $(\mathrm{a}-\mathrm{c})$ small-angle X-ray scattering (SAXS) at different distances, presenting (a) the twodimensional patterns, (b) the one-dimensional profiles revealing the presence of a lamellar ordering $\left(q_{1}\right)$ and two additional broad peaks $\left(q_{2}, q_{3}\right)$, and (c) the temperature-dependent development of the corresponding correlation distances $d_{1}, d_{2}$ and $d_{3}$. All 1D scattering profiles are shifted in intensity on increasing/decreasing temperatures for clarity reasons. Interestingly, when performing POM and SAXS (in capillaries), both systems ANLi-PDMSDGE and ANLi-BDGE show a high affinity to glass surfaces, which is a unique characteristic of TILCs and assigned to the interaction between the glass and the ionic function.[39]

Summarizing these results allows us to propose a peculiar nanostructure for these TLCSICs, produced by the regular organization of the nanoscale building blocks, as schematized in Figure 3. The driving force to nanophase-separated ionophilic/ ionophobic regions, combined with $\pi-\pi$ interactions, results in elementary self-assembled $2 D$ objects (Figure $3 a$ ) with $d_{1}$ being the distance between two naphthalene moieties in the vertical $z$-direction [i.e., the lateral extension of the hydrophobic layer (green)] and $d_{3}$ as the distance between two neighboring naphthalene units along the perpendicular $y$-direction. Due to the shape of the TLCSIC molecules, the ionic functions (blue) are organized out-of-plane with regard to the naphthalene layer (red). The 2D objects are stacked in the third $x$-direction by ionic interactions, with $d_{2}$ as the (temperature-dependent) separation distance between two ionic channels (Figure 3b). Such peculiar nanoscale organization would then indicate preferential 1D $\mathrm{Li}^{+}$conduction pathways along the $\pi-\pi$ stacking direction, decoupled from the lamellar ordering, as it provides the shortest hopping distance for $\mathrm{Li}^{+}$. The regular repetition of the nanoscale building blocks generates per se a complex 3D self-assembled structure as illustrated in Figure 3c.

a)

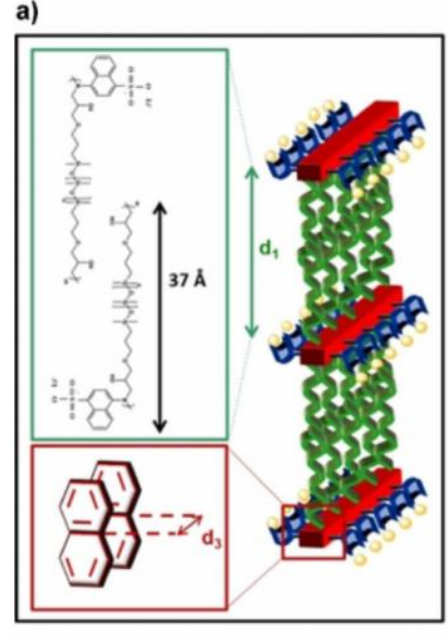

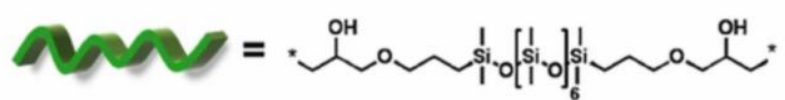

b) c)

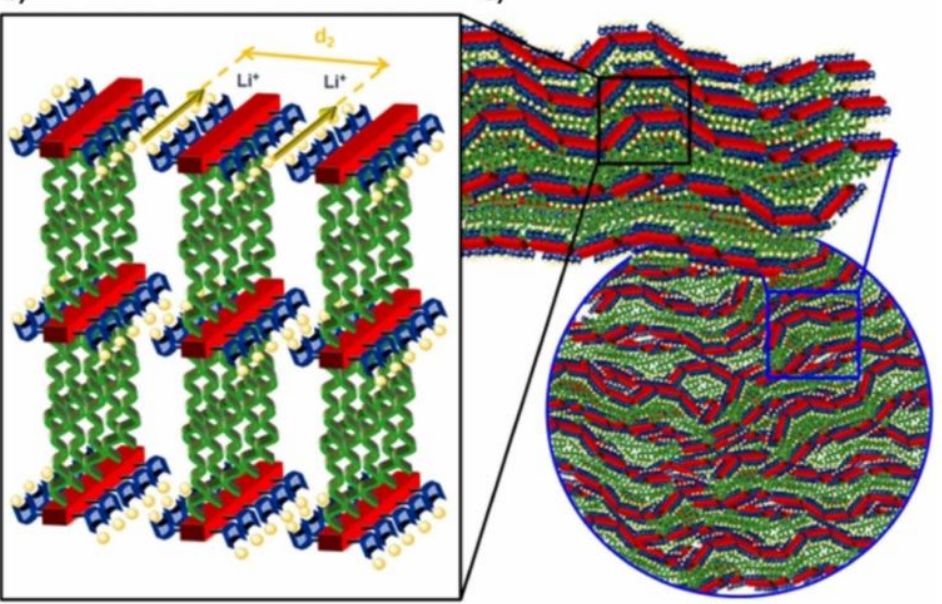

A $=*-\mathrm{SO}_{3}^{-} \mathrm{O}=\mathrm{Li}^{+}$

Figure 3. Illustration of the proposed temperature-induced lamellar alignment of the herein presented thermotropic ionic liquid crystalline single-ion electrolytes, exemplarily giving the distances for ANLi-PDMSDGE, while valid also for ANLiBDGE; the preferred $\mathrm{Li}^{+}$transport pathway along the $\pi-\pi$ stacking direction is indicated by the yellow arrows (b). The chains forming hydrophobic layers are slightly interpenetrated (a). The mesoscale organization is cartooned as a cross-section of the 3D interconnected structure (c). 


\section{Local $\mathrm{Li}^{+}$mobility}

Next, we investigated the local $\mathrm{Li}^{+}$mobility via temperature dependent ${ }^{7} \mathrm{Li}$ nuclear magnetic resonance (NMR) line shape and width measurements for ANLi-BDGE. Figure 4a shows the static ${ }^{7} \mathrm{Li}$ NMR spectra for temperatures between 25 and $170{ }^{\circ} \mathrm{C}$. At low temperatures, the $\mathrm{Li}^{+}$ions are immobile, and the spectra show a broad line with a width of $5 \mathrm{kHz}$, reflecting the different local (spin) environments around the $\mathrm{Li}^{+}$ions/nuclei. The resulting broad distribution of resonance frequencies yields the broad line shape. When the temperature is increased above $50{ }^{\circ} \mathrm{C}$, the onset of $\mathrm{Li}^{+}$mobility with motional correlation rates above $5 \times 10^{3} \mathrm{~s}^{-1}$ and/or the softening of the surrounding structure (see also Figure S4a) result in a temporal averaging of the local environments[32] and, thus, give a clearly narrowed line. The line width variation as a function of temperature is presented in Figure $4 \mathrm{~b}$. The dynamical evolution nicely correlates with the structural reorganization from a disordered low-temperature rubbery phase to the liquid crystalline mesophase above $100{ }^{\circ} \mathrm{C}$, where the formation of long-range ordered ionic channels leads to a more homogeneous chemical environment for the $\mathrm{Li}^{+}$cations. Furthermore, we measured the $\mathrm{Li}$ spin-lattice relaxation rates in the laboratory reference frame $\left(T_{1}{ }^{-1}\right)$ and in the rotating reference frame $\left(T_{1 p^{-1}}\right)$, as plotted in Figure $4 \mathrm{C}$ vs. the inverse temperature. These relaxation rates are sensitive to motional processes on time scales of a few ns and $\mathrm{ms}$, respectively. $T_{1}{ }^{-1}$ shows a monotonic increase with increasing temperature and a soft dynamical transition between the two Arrhenius-type regimes at low temperature $\left(<50^{\circ} \mathrm{C}\right)$ and high temperature $\left(>100^{\circ} \mathrm{C}\right)$, characterized by activation energies of 20 and $12 \mathrm{~kJ} \mathrm{~mol}^{-1}$, respectively. These results strongly support a hopping process across the whole temperature range, originating from the covalent tethering of the anionic function to the rather rigid naphthalene group, which is facilitated in the ordered mesophase, as also reflected in the line width, and consistent with the development of aligned ionic channels (Figure 3). Moreover, $T_{1 \rho^{-1}}$ clearly reveals a maximum at about $100{ }^{\circ} \mathrm{C}$ (i.e., the transition to the mesophase). From this maximum, a hopping rate of about $4 \times 10^{4} \mathrm{~s}^{-1}$ can be roughly estimated for this temperature. This is consistent with the motional narrowing observed in Figure 4a,b. From the low temperature and high-temperature flanks around this maximum an activation energy (Ea) of $0.75^{ \pm 0.01} \mathrm{eV}\left(72.4 \mathrm{kJmol}^{-1}\right)$ can be extracted. This Ea is, as expected, higher than the Ea extracted from $T_{1}^{-1}$ due to the extended timescales probed of up to a few ms, therefore, accounting for additional tortuosity and connectivity effects compared to the local ns dynamics.[32] In conclusion, the NMR results reveal an Arrhenius-type temperature dependency of the Li+ ion dynamics, thus, confirming the $\mathrm{Li}^{+}$conduction mechanism proposed in Figure 3. Importantly, the $\mathrm{Li}^{+}$dynamic clearly correlates to the structural order and transitions observed by POM, DSC, and SAXS, and depend on the probed scale due to the hierarchized geometries/topologies found in this class of soft ionic conductors subjected to a microscale heterogeneity (i.e., the so-called mosaicity) and consisting of interconnected, but randomly oriented highly ordered liquid crystalline nanoscale domains.

a)

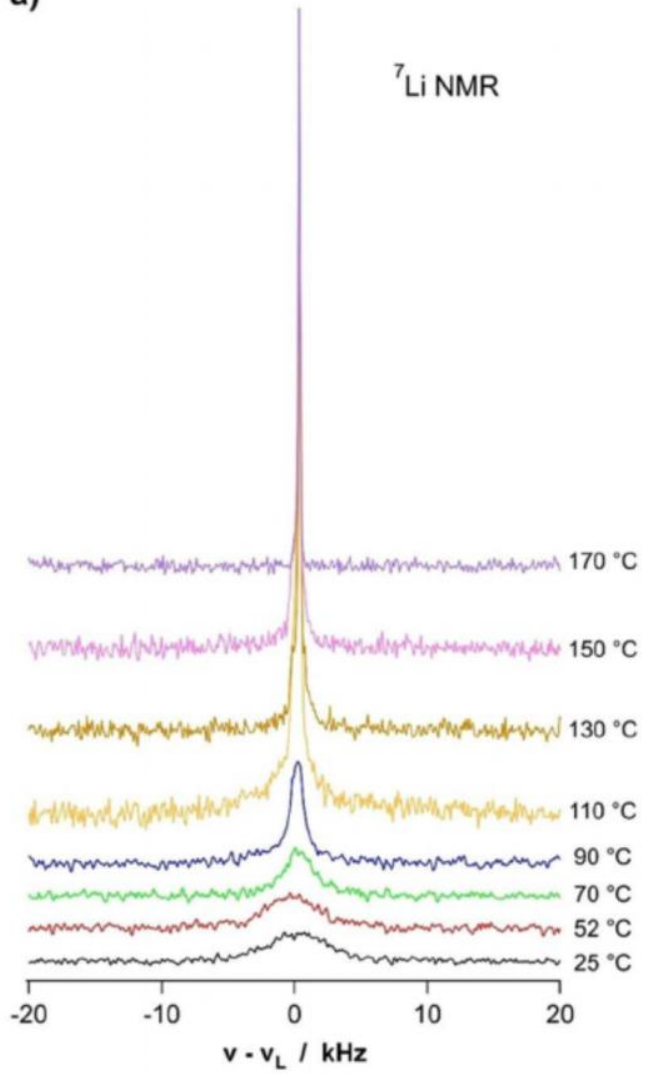

b)
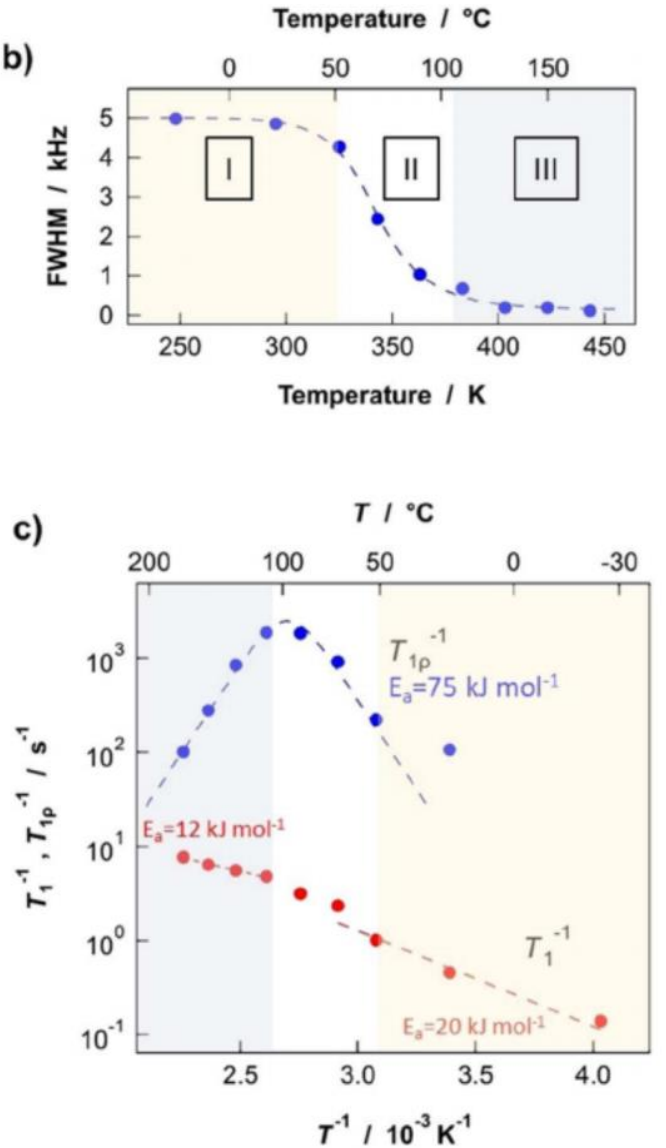

Figure 4. (a) Static ${ }^{7} \mathrm{Li}$ NMR spectra of ANLi-BDGE for temperatures between 25 and $170{ }^{\circ} \mathrm{C}$. (b) The temperature dependence of the line width (full width at half maximum=FWHM). Region I (yellow) and II (white) correspond to the rubbery phase and Region III (grey) to the liquid crystal mesophase. (c) The spin-lattice relaxation rates in the laboratory/rotating reference frame ( $T_{1}{ }^{-1}, T_{1} \rho^{-}$ $\left.{ }^{1}\right)$ versus inverse temperature, including the corresponding activation energies Ea, determined by fitting the $T_{1}^{-1}$ Arrhenius behavior found at low (Region I, yellow) and high (Region III, grey) temperatures as well as the non-monotonic evolution of $T_{1} \rho^{-1}$. 


\section{lonic conductivity and long-range $\mathrm{Li}^{+}$transport}

In a complementary approach to study the $\mathrm{Li}+$ mobility, we determined the ionic conductivity as a function of temperature for ANLi-BDGE and ANLi-PDMSDGE (Figure 5a). Remarkably, the two electrolytes show essentially the same conductivity at $100^{\circ} \mathrm{C}$, indicating that the charge transport at the onset of the mesophase is similarly fast. At further elevated temperatures, however, the conductivity of ANLi-BDGE is increasing more rapidly than for ANLiPDMSDGE, correlating with a significantly higher Ea of $135.8 \mathrm{~kJ} \mathrm{~mol}^{-1} \mathrm{~K}^{-1} \mathrm{vs} .73 .7 \mathrm{~kJ} \mathrm{~mol}^{-1} \mathrm{~K}^{-1}$ for ANLi-PDMSDGE. The $E$ a of ANLi-BDGE is much higher than the values determined by NMR spectroscopy, an effect that, as discussed earlier, originates from the distinct scales probed by the different techniques. Indeed, conductivity measurements integrate additional restrictions to ion mobility due to the initial powdery nature of the TLCSICs and the long-range transport across grain boundaries and large-scale defects. The evolution of the conductivity for both compounds generally reveals an Arrhenius-type temperature-dependency within the mesophase regime, in agreement with the NMR results, corroborating the proposed $\mathrm{Li}+$ hopping mechanism along the $\pi-\pi$ stacking direction (Figure 3 ). For ANLi-BDGE, a clear deviation in slope occurs at temperatures $>180^{\circ} \mathrm{C}$ (i.e., at the transition to the isotropic phase) in fair agreement with the POM results considering the different experimental conditions. Nevertheless, it is also observed that the increase in conductivity (at a logarithmic scale) with temperature within the mesophase regime is not as perfectly linear as in case of ANLi-PDMSDGE. Taking into account also the higher Ea, while the energy for the cationic hopping should be the same (or at least fairly similar) in case of equal jump distances, we assign this difference to the formation of mesoscopic defects in ANLi-BDGE as a result of the different spacer chemistry: While ether-type BDGE can coordinate lithium cations that "leave" the 1D nanochannels at elevated temperatures, creating additional vacancies in the conduction pathway and thus allowing for a higher $\mathrm{Li}^{+}$mobility (potentially accompanied by an additional $\mathrm{Li}^{+}$mobility along the ether functions-at least locally), the siloxane function is less favorable for the formation of such defects. In order to extend the investigation of the $\mathrm{Li}^{+}$mobility (and transport) to the long range, we assembled symmetric Li/ANLi-BDGE/Li cells and applied a constant current of $\pm 1 \mu \mathrm{A}$ (Figure $5 \mathrm{~b}$ ). This current is rather low owing to the relatively low conductivity at $155^{\circ} \mathrm{C}$, which, however, has been the maximum temperature for the utilized experimental setup. Nonetheless, the results unambiguously prove the reversible $\mathrm{Li}^{+}$transport across the ANLi- BDGE electrolyte at an essentially stable $(\sim 7.5 \mathrm{mV})$ and constant overvoltage; as expected for a single-ion conductor with a transference number $\mathrm{t}^{+}$equal to 1.[33] The increase of the open circuit voltage and decrease in voltage hysteresis for the stripping/ plating process is assigned to the reorganization of the lithium/electrolyte interface.[34] Such single-ion conductivity and the resulting homogenous lithium deposition and avoidance of detrimental ion concentration gradients is considered key for the successful realization of lithium metal anodes.[5,35,36] In another experiment, we applied a constant current of $1 \mu \mathrm{A}$ to a Li/ANLi- BDGE/Li cell for $150 \mathrm{~h}$, amounting to a total capacity transfer of $0.15 \mathrm{mAh}$ (Figure 5c). These results confirm the effective lithium transfer and the realization of a stable electrode/electrolyte interface as indicated by the slight decrease in overvoltage before eventually stabilizing at ca. $4 \mathrm{mV}$. This is in good agreement with the stabilizing interface upon reduction, as determined by cyclic voltammetry (Figure S6).
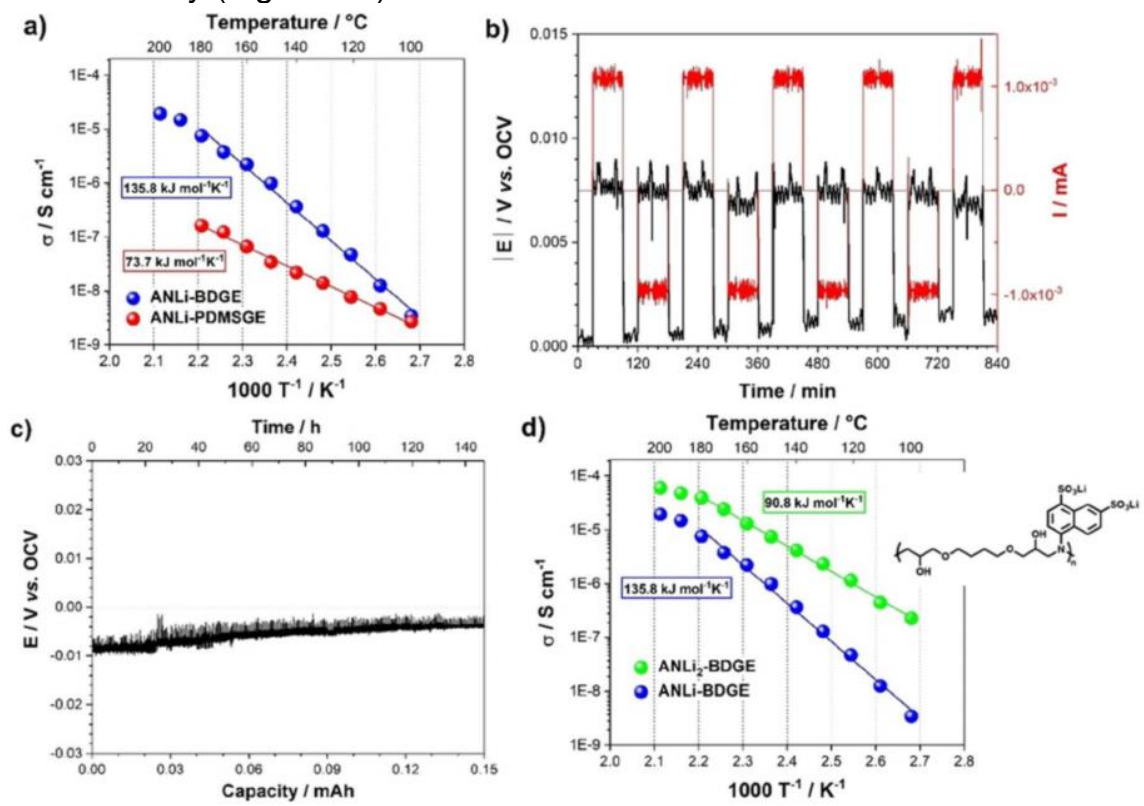

Figure 5. (a) Temperature-dependent ionic conductivity as determined by electrochemical impedance spectroscopy for ANLiBDGE (in blue) and ANLi- PDMSDGE (in red). The corresponding activation energy within the mesomorphic temperature range has been calculated by fitting the experimental data using the Arrhenius equation (see the resulting straight line). (b) Lithium stripping/plating for symmetrical Li/ANLi-BDGE/Li cells at $155^{ \pm 3 \circ} \mathrm{C}$, applying a constant current of $\pm 1 \mu \mathrm{A}\left(\mathrm{ca} .0 .01 \mu \mathrm{A} \mathrm{cm}{ }^{-2}\right.$ ) for 60 $\mathrm{min}$, followed by a $30 \mathrm{~min}$ rest step (electrolyte impregnated into a glass fiber separator; the voltage response has been plotted with a positive sign, independent of the current applied, to allow for a better idea of the overpotential evolution). The fluctuation observed originates from the fluctuating current. (c) Lithium "stripping" experiment for a symmetrical Li/ANLi-BDGE/Li cell applying a constant current of $1 \mu \mathrm{A}$ (ca. $0.01 \mu \mathrm{A} \mathrm{cm}{ }^{-2}$ ) for $150 \mathrm{~h}$ at $155^{\circ} \mathrm{C}$ (each $30 \mathrm{~min}$, there is a 5 min rest step; no separator). (d) Temperature-dependent ionic conductivity for ANLi2-BDGE (in green; see also the chemical formula as inset) in comparison with ANLi-BDGE (in blue). The corresponding activation energies within the mesomorphic temperature range have been calculated by fitting the experimental data using the Arrhenius equation. 
In an attempt to increase the ionic conductivity, especially at lower temperatures, we considered the general strategies for solid-state electrolytes, including (i) the realization of long range orientation (e.g., via external stimuli like mechanical shearing[24] or applying an electric field),[37] (ii) a facilitated ion dissociation (e.g., by further delocalizing the negative charge), (iii) the insertion and control of (artificial) defects,[38] or (iv) an increase of the charge carrier density. As a first approach, we chose the latter strategy and introduced a second ionic function onto the naphthalene moiety (Figure $5 \mathrm{~d}$ ). This approach proved to be successful and we achieved a substantial increase in conductivity by ca. one and three $\operatorname{order}(\mathrm{s})$ of magnitude at medium and relatively low temperatures, respectively, approaching a conductivity of $10^{-4} \mathrm{~S} \mathrm{~cm}^{-1}$ at elevated temperatures. With respect to the substantially lower activation energy of $90.8 \mathrm{~kJ} \mathrm{~mol}^{-1} \mathrm{~K}^{-1}$ (i.e., a decrease by about one-third), we assign this substantial improvement to a facilitated ion hopping, originating from the higher ion dissociation due to the stabilizing effect of the second ionic function. Additionally, the introduction of the second ionic function appears to reduce the formation of defects; in fact, the temperature-dependency of the ionic conductivity is clearly Arrhenius-like.

\section{Conclusions}

We have shown that the tailored molecular design of organic liquid-crystalline Li+ electrolytes may enable decoupling of segmental relaxation and charge transport and, thus, the realization of solid-like Li+ conduction along well-defined nanochannels like in crystalline inorganic electrolytes. While the local-scale activation energy is already in a suitable range for practical applications, the long-range conductivity remains limited to elevated temperatures at this point. However, we anticipate that a substantial gain in ionic conductivity may be obtained by applying (one or a combination of) the abovementioned strategies. The ultimate goal could be the realization of a new generation of smart electrolytes showing anisotropic ion conduction with an enhanced alignment under an electric field following the dis-/charge "direction" of the cell and becoming an insulator when the cell is left to rest.

\section{Acknowledgements}

This work was funded by the NanoSciences Programme (CEA) within the framework of the ELLIPSE project. D.B. would like to acknowledge moreover the EU/CEA Enhanced Eurotalents Fellowship for financial support. All authors would like to warmly thank Dr. Alain Farchi, Dr. Arnaud de Geyer, and Dr. Cyrille Rochas for assistance and experimental support with the SAXS experiments

performed at CEA-IRIG-DEPHY-MEM-SGX and at the ESRF (beamline BM02-D2AM), respectively. In addition, the authors would like to acknowledge the European Synchrotron Radiation Facility (ESRF) for granting beam-time to perform part of the herein reported SAXS experiments (under the proposal $\mathrm{N}^{\circ} 02-01-854$ ).

\section{Conflict of Interest}

The authors declare no conflict of interest.

\section{References}

[1] S. Chu, A. Majumdar, Nature 2012, 488, 294.

[2] N. Kittner, F. Lill, D. M. Kammen, Nat. Energy 2017, 2, 17125.

[3] Z. P. Cano, D. Banham, S. Ye, A. Hintennach, J. Lu, M. Fowler, Z. Chen, Nat. Energy 2018, 3, 279.

[4] A. Varzi, R. Raccichini, S. Passerini, B. Scrosati, J. Mater. Chem. A 2016, 4,17251.

[5] D. T. Hallinan, N. P. Balsara, Annu. Rev. Mater. Res. 2013, 43, 503.

[6] D. Bresser, K. Hosoi, D. Howell, H. Li, H. Zeisel, K. Amine, S. Passerini, J. Power Sources 2018, $382,176$.

[7] M. Marinaro, D. Bresser, E. Beyer, P. Faguy, K. Hosoi, H. Li, J. Sakovica, K. Amine, M. Wohlfahrt-Mehrens, S. Passerini, J. Power Sources 2020, 459, 228073.

[8] M. Armand, Adv. Mater. 1990, 2, 278.

[9] M. A. Ratner, P. Johansson, D. F. Shriver, MRS Bull. 2000, 25, 31.

[10] X. Wei, D. F. Shriver, Chem. Mater. 1998, 10, 2307.

[11] D. Golodnitsky, E. Strauss, E. Peled, S. Greenbaum, J. Electrochem. Soc. 2015, 162, A2551.

[12] C. A. Angell, C. Liu, E. Sanchez, Nature 1993, 362, 137.

[13] D. Bresser, S. Lyonnard, C. lojoiu, L. Picard, S. Passerini, Mol. Syst. Des.Eng. 2019, 4, 779.

[14] V. Bocharova, A. P. Sokolov, Macromolecules 2020, 53, 4141.

[15] Z. Gadjourova, Y. G. Andreev, D. P. Tunstall, P. G. Bruce, Nature 2001,412, 520.

[16] S. Cheng, D. M. Smith, C. Y. Li, Macromolecules 2014, 47, 3978.

[17] Y. Wang, A. L. Agapov, F. Fan, K. Hong, X. Yu, J. Mays, A. P. Sokolov, Phys. Rev. Lett. 2012, $108,088303$.

[18] V. Delhorbe, D. Bresser, H. Mendil-Jakani, P. Rannou, L. Bernard, T. Gutel, S. Lyonnard, L. Picard, Macromolecules 2017, $50,4309$.

[19] F. B. Dias, J. P. Voss, S. V. Batty, P. V. Wright, G. Ungar, Macromol. Rapid Commun. 1994, $15,961$.

[20] G. S. McHattie, C. T. Imrie, M. D. Ingram, Electrochim. Acta 1998, 43, 1151.

[21] R. L. Kerr, S. A. Miller, R. K. Shoemaker, B. J. Elliott, D. L. Gin, J. Am. Chem. Soc. 2009, 131, 15972.

[22] T. Kato, M. Yoshio, T. Ichikawa, B. Soberats, H. Ohno, M. Funahashi, Nat. Rev. Mater. 2017, 2, 17001.

[23] T. Onuma, E. Hosono, M. Takenouchi, J. Sakuda, S. Kajiyama, M. Yoshio, T. Kato, ACS Omega 2018, 3, 159.

[24] F. B. Dias, S. V. Batty, A. Gupta, G. Ungar, J. P. Voss, P. V. Wright, Electrochim. Acta 1998, 43, 1217.

[25] H. Shao, T. Nguyen, N. C. Romano, D. A. Modarelli, J. R. Parquette, J. Am. Chem. Soc. 2009, $131,16374$.

[26] F. B. Dias, S. V. Batty, G. Ungar, J. P. Voss, P. V. Wright, J. Chem. Soc. Faraday Trans. 1996, 92, 2599.

[27] K. Binnemans, Chem. Rev. 2005, 105, 4148.

[28] K. Goossens, K. Lava, C. W. Bielawski, K. Binnemans, Chem. Rev. 2016, 116, 4643.

[29] T. Kato, J. M. J. Frechet, J. Am. Chem. Soc. 1989, 111, 8533.

[30] I. M. Khan, Y. Yuan, D. Fish, E. Wu, J. Smid, Macromolecules 1988, 21, 2684.

[31] J. Kalhoff, G. G. Eshetu, D. Bresser, S. Passerini, ChemSusChem 2015, 8, 2154.

[32] P. Heitjans, S. Indris, J. Phys. Condens. Matter 2003, 15, R1257.

[33] P. G. Bruce, C. A. Vincent, Solid State lonics 1990, 40-41, 607. 
[34] H.-D. Nguyen, G.-T. Kim, J. Shi, E. Paillard, P. Judeinstein, S. Lyonnard, D. Bresser, C. lojoiu, Energy Environ. Sci. 2018, $11,3298$.

[35] H. Zhang, C. Li, M. Piszcz, E. Coya, T. Rojo, L. M. Rodriguez-Martinez, M. Armand, Z. Zhou, Chem. Soc. Rev. $2017,46,797$.

[36] C. Brissot, M. Rosso, J.-N. Chazalviel, S. Lascaud, J. Electrochem. Soc. 1999, 146, 4393.

[37] H. Shimura, M. Yoshio, A. Hamasaki, T. Mukai, H. Ohno, T. Kato, Adv. Mater. 2009, 21, 1591.

[38] A. M. Christie, S. J. Lilley, E. Staunton, Y. G. Andreev, P. G. Bruce, Nature 2005, 433, 50.

[39] S. Ujiie, K. limura, Macromolecules 1992, 25, 3174.

Manuscript received: August 21, 2020

Revised manuscript received: September 7, 2020

Accepted manuscript online: September 18, 2020

Version of record online: October 13, 2020 